\title{
Preparation of Derivatives of Betulinic Acid, Steviol and Isosteviol and Evaluation of Antitrypanosomal and Antimalarial Activities
}

\author{
Asad Ullah, ${ }^{a}$ Leopoldo C. Baratto, ${ }^{a}$ Renata C. Paula,${ }^{b}$ Luz Helena V. Silva, ${ }^{c}$ \\ Maurilio J. Soares ${ }^{c}$ and Brás H. Oliveira*a \\ ${ }^{a}$ Departamento de Química, UFPR, Centro Politécnico, CP 19081, 81531-970 Curitiba-PR, Brazil \\ ${ }^{b}$ Faculdade de Farmácia, UFMG, Av. Presidente Antônio Carlos, 6627, \\ 31270-901 Belo Horizonte-MG, Brazil \\ ${ }^{c}$ Laboratório de Biologia Celular, Instituto Carlos Chagas, Fiocruz, Curitiba-PR, Brazil
}

\begin{abstract}
Derivatives of steviol, isosteviol and betulinic acid were prepared and tested in vitro against Plasmodium falciparum W2 (chloroquine-resistant) and Trypanosoma cruzi. The best results against P. falciparum were found for the betulinic acid derivatives 3-[(2,4-dinitrophenyl)hydrazono]lup20(29)-en-28-oic acid and 3-hydroxy-20-[(2,4-dinitrophenyl)hydrazono]-29-norlupan-28-oic acid (values concentration in that the growth of the parasites is $50 \%$ inhibited, $\mathrm{IC}_{50}=9.96,6.45$ and $21.06 \mu \mathrm{g} \mathrm{mL}^{-1}$, respectively). Isosteviol was inactive but its 4-nitro- and 2,4-dinitrophenylhydrazone showed moderate activity $\left(\mathrm{IC}_{50}=17.50-22.58 \mu \mathrm{g} \mathrm{mL} \mathrm{m}^{-1}\right)$. The derivatives of betulinic acid were more selective to the parasite when compared with hepatoblastoma HepG2 A16 cells. The results showed that nitrophenylhydrazone derivatives of lupane and beyerane terpenes are promising prototypes of antimalarial agents. Among the compounds tested against $T$. cruzi the oxime of isosteviol was active against both epimastigote and trypomastigote forms of the parasite.
\end{abstract}

Keywords: betulinic acid, steviol, isosteviol, Trypanosoma cruzi, Plasmodium falciparum

\section{Introduction}

Malaria is a disease of worldwide importance caused by the parasite Plasmodium falciparum. A report from the World Health Organization estimates that 207 million cases and 607,000 deaths occurred in 2012. ${ }^{1}$ In Brazil more than 267,000 cases were reported in $2011 .^{2}$ Moreover, the resistance of $P$. falciparum to drugs, like the classical cloroquine and also the newer artemisinin and derivatives, has increased. ${ }^{3}$ The rise of resistant strains of the parasite motivates the search for new and more effective drugs.

Natural products have also been an important source of antimalarial drugs. Atovaquone (1a), quinine (1b), artemisinin (1c, Figure 1) and semi-synthetic derivatives have been used for the treatment of the disease. ${ }^{4}$ Many others have also been described as active in vitro against the parasite and could become lead compounds with new mechanisms of action in order to overcome resistance. ${ }^{5}$ Triterpene-rich plant extracts containing betulinic acid (2a, Figure 2) have been reported as antiplasmodial. Initial evidences of the activity

*e-mail: bho@ufpr.br of betulinic acid indicated a moderately action against P. falciparum (NF 54) with $\mathrm{IC}_{50}$ values (concentration in that the growth of the parasites is $50 \%$ inhibited) $=10.5 \mu \mathrm{g} \mathrm{mL}^{-1}$. $^{6}$ Other studies have also reported the antimalarial potential of that compound and its derivatives. ${ }^{7-20} \mathrm{~A}$ few tetracyclic diterpenes have also shown antimalarial properties. One example is a norpimaran lactone (1d, Figure 1) obtained from manool, ${ }^{21}$ and another is kaurenoic acid (3a, Figure 3) described as weakly active. ${ }^{22}$ Lactone kaurane diterpenoids (1e, Figure 1), isolated from Parinari capensis, have shown strong in vitro antimalarial activity, but they were highly toxic to human kidney epithelial cells. ${ }^{23}$

Chagas disease is another serious chronic illness caused by Trypanosoma cruzi, with most infected people living in Latin America countries. It is estimated that 7-8 million people are infected worldwide with Chagas disease. ${ }^{24}$ Benznidazole has been used to treat Chagas disease in both acute and initial chronic phases. ${ }^{25}$ However, this drug presents serious side effects is not totally effective development of resistance. ${ }^{26}$ Therefore the search for alternative drugs is necessary.

Kaurenes (e.g., steviol, 3c, Figure 3) and beyerenes (e.g., isosteviol, 4a, Scheme 1) are tetracyclic diterpenes 
<smiles>O=C1C2CCCCC2C(=O)C([C@H]2CC[C@H](c3ccc(O)cc3)CC2)C1O</smiles><smiles>C=CC1CN2CCC1C[C@H]2[C@H](O)c1ccnc2ccc(OC)cc12</smiles><smiles>C[C@H]1CC[C@H]2[C@@H](C)C(=O)O[C@@H]3O[C@]4(C)CCC1[C@@]32OO4</smiles>

$1 \mathrm{~b}$

1c<smiles></smiles><smiles>[R]OOOC</smiles>

Figure 1. Structures of antimalarial natural products.

with similar carbon backbones, except for rings $\mathrm{C}$ and $\mathrm{D}$ due to the inversion of stereochemistry at C-8 and C-13. Some kaurenes have been described as active against T. cruzi. One report describes significant in vitro and in vivo activities of the natural compounds kaurenol and kaurenoic acid. ${ }^{27}$ Derivatives of kaurenoic acid were prepared and methyl ent-16Z-oxime-17-norkauran-19-oate showed improved activity. ${ }^{28}$ Another natural ent-9a-hydroxy-15bE-cinnamoyloxy-16-kauren-19-oic acid was also active in vitro. ${ }^{29}$ Thiosemicarbazones derivatives of kaurenoic acid were also prepared and some showed increased activity; the $o$-nitrobenzaldehyde-thiosemicarbazone derivative was the most active compound with $\mathrm{IC}_{50}$ of $2.0 \mathrm{mM} .^{30}$
In this report we describe the preparation and evaluation of the antimalarial and antitrypanosomal activities of known and new derivatives of betulinic acid and some kaurene and beyerene tetracyclic diterpenoids.

\section{Results and Discussion}

\section{Chemistry}

The structures of all compounds prepared are shown in Figures 1, 2 and 3. The known compounds were obtained as previously reported (see Experimental section). The $p$-methoxyphenacyl esters of steviol and
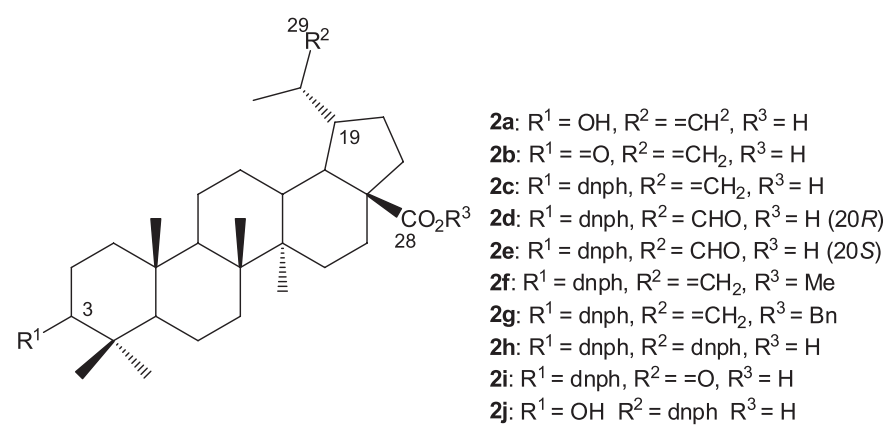

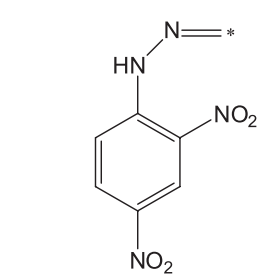

2,4-Dinitrophenylhydrazone (dnph)

Figure 2. Structures of betulinic acid (2a) and derivatives.

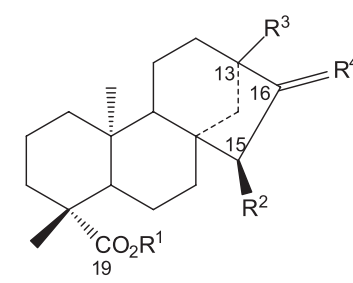

3a: $\mathrm{R}^{1}=\mathrm{CO}_{2} \mathrm{H}, \mathrm{R}^{2}=\mathrm{R}^{3}=\mathrm{H}, \mathrm{R}^{4}=\mathrm{CH}_{2}$ 3b: $\mathrm{R}^{1}=\mathrm{CO}_{2} \mathrm{H}, \mathrm{R}^{3}=\mathrm{H}, \mathrm{R}^{2}=\mathrm{OAc}, \mathrm{R}^{4}=\mathrm{CH}_{2}$ 3c: $\mathrm{R}^{1}=\mathrm{CO}_{2} \mathrm{H}, \mathrm{R}^{2}=\mathrm{H}, \mathrm{R}^{3}=\mathrm{OH}, \mathrm{R}^{4}=\mathrm{CH}_{2}$ 3d: $\mathrm{R}^{1}=\mathrm{CO}_{2} \mathrm{H}, \mathrm{R}^{2}=\mathrm{H}, \mathrm{R}^{3}=\mathrm{OH}, 16,17$-epoxy 3e: $\mathrm{R}^{1}=\mathrm{CO}_{2}-\mathrm{pmp}, \mathrm{R}^{2}=\mathrm{H}, \mathrm{R}^{3}=\mathrm{OH}, \mathrm{R}^{4}=\mathrm{CH}_{2}$ 3f: $\mathrm{R}^{1}=\mathrm{CO}_{2}$-glucosyl, $\mathrm{R}^{2}=\mathrm{H}, \mathrm{R}^{3}=$ sophorosyl, $\mathrm{R}^{4}=\mathrm{CH}_{2}$ 3f: $\mathrm{R}^{1}=\mathrm{CO}_{2} \mathrm{CH}_{3}, \mathrm{R}^{2}=\mathrm{R}^{3}=\mathrm{H}, \mathrm{R}^{4}=\mathrm{NOH}$ 3g: $\mathrm{R}^{1}=\mathrm{CH}_{2} \mathrm{OH}, \mathrm{R}^{2}=\mathrm{R}^{3}=\mathrm{H}, \mathrm{R}^{4}=\mathrm{NOH}$<smiles>COc1ccc(C(C)=O)cc1</smiles>

p-Methoxyphenacyl (pmp)

Figure 3. Structures of steviol (3c) and derivatives. 
<smiles>CC12CCC3C(CC1=NO)[C@]1(C)CCCCC31CC(=O)[C@]2(C)C(=O)O</smiles><smiles></smiles><smiles>C=C1CC1C</smiles>

40<smiles>CC1(C)CCC2C(CC(=O)O)(CCC3[C@@](C)(C(=O)O)CCC[C@@]23C)C1</smiles><smiles>C[C@H]1CC[C@H]1C</smiles><smiles>COC(=O)[C@]1(C)CCC[C@@]2(C)CC3(CC1)CC(C)(C(=N)N)CCC32</smiles><smiles>CC12CCC3C4(CCC(C(=O)O)[C@@]4(C)CCC[C@@]3(C)C1)CC2=O</smiles><smiles>C=C[C+]C</smiles><smiles>CC12CCC3C(CCC4[C@@](C)(C(=O)O)CCC[C@@]34C)(CC1=O)C2</smiles>

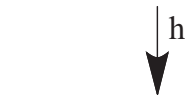

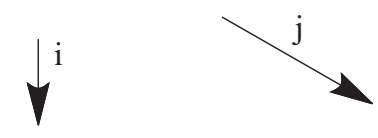

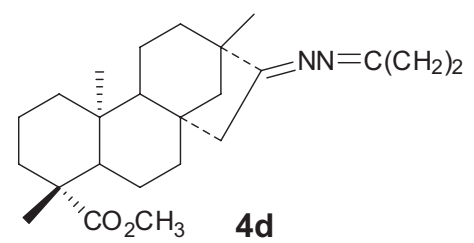

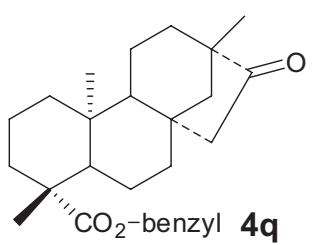<smiles>[R]C([R]([H])c1ccccc1)=[R]([H])[H]</smiles>

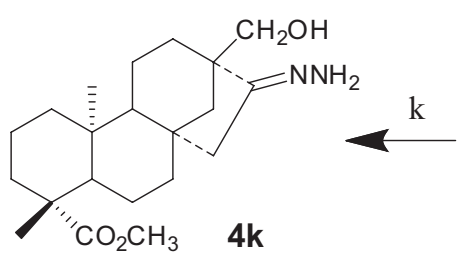<smiles>CC1(CO)CC23CCC([C@]4(C)CCC[C@@]4(C)I)C2(CC[C@](C)(C(=O)O)C3)CC1=O</smiles><smiles>[R]C1=CC23CCC4[C@@](C)(CCC[C@@]4(C)C(=O)O)C2CCC1(CO)C3</smiles><smiles>C[C@]12CCC[C@]1(C)C1CCC3(CO)CC(O)C(CCC13)C2</smiles><smiles>C[14CH3]</smiles>

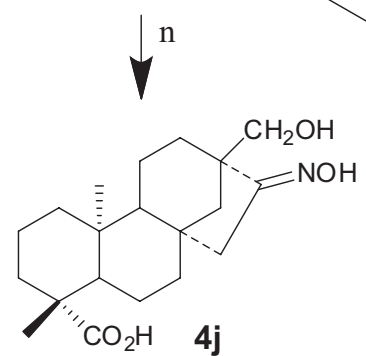

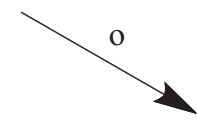

4I, $\mathrm{R}=\mathrm{nph}$

4m, R = dnph<smiles>C[C@]1(C(=O)O)CCC[C@]2(C)C3CCC(CO)(CC3=N)CC23CCCC[C@]31C</smiles>

Scheme 1. Preparation of isosteviol derivatives. $(\mathrm{a}, \mathrm{b}) \mathrm{p}$-methoxyphenacyl bromide, acetone, triethylamine, microwave, 4 min, $(\mathbf{4 o} 78 \%, \mathbf{4 p} 75 \%)$; (c) hydroxylamine chloride, sodium acetate, water, $\mathrm{EtOH}$, room temperature (rt), $24 \mathrm{~h}\left(78 \%\right.$ ); (d) $\mathrm{NaBH}_{4}, \mathrm{EtOH}, \mathrm{rt}, 1 \mathrm{~h}, 80 \%$; (e) $\mathrm{CH}_{2} \mathrm{~N}_{2}$, ethyl ether, $\mathrm{MeOH}$; (f) hydrazine hydrate, $\mathrm{MeOH}$, reflux, $8 \mathrm{~h}, 83 \%$; (g) p-methoxyphenacyl bromide, acetone, triethylamine, microwave, $4 \mathrm{~min}, 81 \%$; (h) acetone, reflux, 2 h, 83\%; (i) benzyl chloride, acetone, $\mathrm{K}_{2} \mathrm{CO}_{3}$, reflux, $2 \mathrm{~h}, 83 \%$; (j, l) nitrophenylhydrazine (or 2,4-dinitrophenylhydrazine), $\mathrm{EtOH}_{2} \mathrm{H}_{2} \mathrm{O}, \mathrm{H}_{2} \mathrm{SO}_{4}$, rt, 12 h (4e 51\%, 4f 70\%, 4l 49\%, 4m 57\%); (k) hydrazine hydrate, MeOH, reflux, 2 h, 86\%; (o) p-methoxyphenacyl bromide, acetone, triethylamine, microwave, 4 min, 78\%; (m) $\mathrm{NaBH}_{4}$, EtOH, rt, 1 h, 80\%; (n) hydroxylamine chloride, sodium acetate, water, EtOH, rt, 24 h (68\%); (o) hydrazine hydrate, $\mathrm{MeOH}$, reflux, 8 h, 86\%; pmp = p-methoxyphenacyl; $\mathrm{nph}$ = 4-nitrophenylhydrazone; dnph = 2,4-dinitrophenylhydrazone. 
isosteviol were obtained by reaction with 2-bromo4'-methoxyacetophenone with microwave irradiation, which allowed a short reaction time (ca. 5 minutes). The ${ }^{13} \mathrm{C}$ nuclear magnetic resonance (NMR) data showed the characteristic signals for the methoxyphenacyl moiety e.g., the ketone (ca. $190 \mathrm{ppm}$ ) and methoxy (ca. $65 \mathrm{ppm}$ ) groups.

The hydrazones were obtained in the usual fashion by reaction of the carbonyl group (C16) with selected hydrazines. The ${ }^{13} \mathrm{C}$ NMR data showed the characteristic signal of $\mathrm{C}=\mathrm{N}$ group at $162-174 \mathrm{ppm}$. The other spectroscopic data mass spectroscopy, infrared (MS, IR) were also compatible with respective structures.

\section{Bioassays}

\section{Anti $P$. falciparum}

The results of growth inhibition of the test compounds on the chloroquine-resistant strain P. falciparum W2 are shown on Table 1 (the inactive and slightly active were omitted). The activity of betulinic acid (2a) was confirmed and most of the modifications in its molecular structure did not increased activity significantly. Oxidation at C-3 to betulonic acid (2b) drastically reduced the activity. Attachment of a 2,4-dinitrophenylhydrazone (dnph) moiety at either C-3 (2c) or C-29 (2j) increased activity two and three fold relative to parent compound, respectively (Table 1). However, the derivative containing the same substituent at both positions (2h) was less active than betulinic acid (2a). Esterification at C-28 with either methyl (2f) or benzyl (2g) or modifications at the isopropenyl group at C-19 (2d, 2e, 2i) decreased the activity of the 2,4-dnph derivative (2c). Therefore, a free carboxyl at C-28, hydroxyl at C-3 and an isopropenyl at C-19 are important for the activity of these 2,4-dnph derivatives of betulinic acid.

Table 1. $\mathrm{IC}_{50}$ of the most active compounds against $P$. falciparum $\mathrm{W} 2$

\begin{tabular}{lcc}
\hline Compound & $\mathrm{IC}_{50}{ }^{\mathrm{a}} /\left(\mu \mathrm{g} \mathrm{mL}^{-1}\right)$ & Classification \\
\hline $\mathbf{2 a}$ & $17.7 \pm 1.3$ & moderately active \\
$\mathbf{2 c}$ & $9.96 \pm 1.20$ & active \\
$\mathbf{2} \mathbf{j}$ & $6.45 \pm 1.47$ & active \\
$\mathbf{4 e}$ & $17.50 \pm 4.83$ & moderately active \\
$\mathbf{4 f}$ & $21.06 \pm 3.15$ & moderately active \\
$\mathbf{4}$ & $22.58 \pm 4.92$ & moderately active \\
$\mathbf{4}$ & $18.80 \pm 6.00$ & moderately active \\
Chloroquine & $0.085 \pm 0.0201$ & very active \\
\hline${ }^{a} \mathrm{IC}_{50}:$ concentration in that the growth of the parasites is 50\% inhibited.
\end{tabular}

An important aspect of drug candidates is their selectivity. Therefore, the most active compounds, $\mathbf{2 c}$ and $\mathbf{2 j}$, were tested against HepG2 A16 cells, considered as hepatic cytotoxicity markers. ${ }^{31}$ The results (Table 2 ) showed they were non-toxic, and the selectivity index indicated that both derivatives are at least 100 times more selective against $P$. falciparum.

Table 2. $\mathrm{CC}_{50}\left(\mu \mathrm{g} \mathrm{mL}^{-1}\right)$ of $\mathbf{2 c}$ and $\mathbf{2} \mathbf{j}$ against HepG2 A16 cells and respective selectivity indexes (SI)

\begin{tabular}{lcc}
\hline Compound & $\mathrm{CC}_{50}{ }^{a} /\left(\mu \mathrm{g} \mathrm{mL}^{-1}\right)$ & Selectivity index $(\mathrm{SI})$ \\
\hline $\mathbf{2 c}$ & $>1000$ & 100.40 \\
$\mathbf{2 j}$ & $>1000$ & 155.03 \\
Chloroquine & 193.59 & $2,277.52$ \\
\hline
\end{tabular}

${ }^{\mathrm{a}} \mathrm{CC}_{50}$ : cytotoxic concentration that inhibit $50 \%$ the cell growth.

Some kaurenes, like kaurenoic acid (3a), have been described as weakly antiplasmodial, ${ }^{22}$ whereas some kaurene lactones (Figure 1) were very active but highly toxic against human epithelial kidney cells. ${ }^{23}$ Xylopic acid (3b), on the other hand, has been described as antimicrobial. ${ }^{32}$ Our results showed that kaurenoic acid (3a), xylopic acid (3b) and steviol (3c) were inactive (data not shown). The results of the beyerene compounds showed that all modifications of the inactive isosteviol (4a) led to moderately active derivatives. The most actives were 4-nitrophenyl (4e, 4l) or 2,4-dinitrophenylhydrazone (4f, 4m) derivatives. The other hydrazones were inactive. Comparing the results for the two classes of diterpenes, those with beyerene backbone displayed the best activities.

\section{Anti T. cruzi}

Some compounds were also tested against epimastigotes and trypomastigotes forms of T. cruzi. The results (Table 3 ) shows that most compounds were inactive to the parasite. The oxime of isosteviol (4b), however, showed significant activity against both forms of the parasite. The same compound, on the other hand, did not show activity against $P$. falciparum, indicating species selectivity.

Similar oximes have shown activity in vitro against T. cruzi. The oximes $\mathbf{3 f}$ and $\mathbf{3 g}$, obtained from kaurenoic acid (3a) showed significant activity. ${ }^{28}$ Oximes of tetracyclic diterpenoids are, therefore, important leads for development of anti-malarial compounds. Interestingly, the same compound was inactive against $P$. falciparum.

\section{Conclusions}

Our results showed that the antiplasmodial activity of tetracyclic diterpenes and triterpenes may be increased by structural modifications. A total of 33 compounds (10 derivatives of betulinic acid, 6 kauranes and 17 beyeranes) were prepared and tested in vitro against $P$. falciparum. The addition of a nitrophenylhydrazone moiety increased 
Table 3. In vitro biological activity of isosteviol derivatives on $T$. cruzi epimastigotes, trypomastigotes $\left(\mathrm{IC}_{50}\right)$ and Vero cells $\left(\mathrm{CC}_{50}\right)$ and corresponding selective index (SI)

\begin{tabular}{lccc}
\hline Compound & $\begin{array}{c}\mathrm{IC}_{50}, 24 \mathrm{~h} / \\
\mu \mathrm{M}\end{array}$ & $\begin{array}{c}\mathrm{CC}_{50}, 24 \mathrm{~h} / \\
\mu \mathrm{M}\end{array}$ & $\begin{array}{c}\text { Selective index } \\
(\mathrm{SI})\end{array}$ \\
\hline 3a & $>700$ & $\mathrm{ND}$ & \\
$\mathbf{3 d}$ & $>1400$ & $\mathrm{ND}$ & \\
$\mathbf{3 e}$ & $>2300$ & $\mathrm{ND}$ & \\
$\mathbf{4 a}$ & $>500$ & $\mathrm{ND}$ & \\
$\mathbf{4 b}$ & 167.9 & 135.44 & 0.81 \\
$\mathbf{4 f}$ & $>400$ & $\mathrm{ND}$ & \\
$\mathbf{4 g}$ & $>18,000$ & $\mathrm{ND}$ & \\
$\mathbf{4 n}$ & $>400$ & $\mathrm{ND}$ & \\
$\mathbf{4 q}$ & $>600$ & $\mathrm{ND}$ & \\
Benznidazole & 45 & 3954.3 \\
\hline \multicolumn{4}{c}{ On cell-derived Trypomastigotes } \\
\hline 4b & 106.08 & 135.44 \\
Benznidazole & 61.8 & 3924.3 \\
\hline
\end{tabular}

ND: not done; $\mathrm{IC}_{50}$ : concentration in that the growth of the parasites is $50 \%$ inhibited; $\mathrm{CC}_{50}$ : cytotoxic concentration that inhibit $50 \%$ the cell growth.

significantly the activity. Some beyerene derivatives were moderately actives and the best results were obtained for the derivatives of betulinic acid, which were also very selective. The activity of the oxime of isosteviol (4b) against T. cruzi, and its inactivity against $P$. falciparum, indicates that appropriate modification of these compounds may result in species selective activities. These classes of terpenes, therefore, are promising models for the development of new drugs for the treatment of malaria and Chagas disease.

\section{Experimental}

\section{General experimental details}

IR and NMR spectra were obtained with a Bio-Rad (FTS3500GX) and Bruker (Avance DRX400 or DPX 200) spectrometers, respectively. Mass spectra electrospray ionisation (ESI) were acquired with a Thermo Fisher Scientific Inc. (LTQ XL Linear Ion Trap). Planar centrifugal chromatography was performed with a Chromatotron (Harrison Research) model 7924T, using silica-gel PF254 (Merck, art. No.7749). Thin-layer chromatography (TLC) for reaction monitoring was performed on silica plates from Merck (art. No. 5554). Solvents were distilled before use.

Isolation of betulinic acid and steviol

Betulinic acid and derivatives (2a-2j) were prepared as previously described. ${ }^{33}$ Kaurenoic acid (3a) was isolated from Annona glabra ${ }^{34}$ and xylopic acid (3b) was isolated from Xylopia frutescens. ${ }^{35}$ Steviol (3c) was obtained from stevioside (3f) by reaction with $\mathrm{NaIO}_{4}$ followed by treatment with base. ${ }^{36}$ Isosteviol $(\mathbf{4 a})^{37}$ and its derivatives 16-hydroxyisosteviol (4g), ${ }^{38}$ isosteviol dnph (4f),${ }^{39}$ and isosteviol oxime $(\mathbf{4 b})^{40}$ were prepared as described previously.

General procedure for the preparation of $p$-methoxyphenacyl esters

Steviol or isosteviol derivative $(0.31 \mathrm{mmol})$ was added to a mixture of 2-bromo-4'-methoxyacetophenone (225 mg, $0.98 \mathrm{mmol})$ and triethylamine $\left(20 \mathrm{mg} \mathrm{mL}^{-1}\right.$ in acetone $)$ and the mixture was irradiated in a microwave oven. ${ }^{41}$ After reaction completion (ca. 4 minutes) acetic acid $(40 \mu \mathrm{L})$ was added and the mixture was irradiated again for 1 minute. The mixture was then chromatographed on a Chromatotron using silica rotors $(1 \mathrm{~mm})$, and elution was made with appropriate mixtures of acetone: $n$-hexane. The product obtained was then characterized by spectroscopic methods.

\section{Steviol $p$-methoxyphenacyl ester (3e)}

White solid; yield 78\%; IR (KBr) $v / \mathrm{cm}^{-1} 3490,2986$, 1728, 1689, 1602, 1145; ${ }^{1} \mathrm{H}$ NMR $\left(200 \mathrm{MHz}, \mathrm{CDCl}_{3}\right)$ 7.90 (d, 2H, J 8.9 Hz, H2', H6'), $\delta 6.95$ (d, 2H, J 8.9 Hz, H3', H5'), $\delta 5.27$ (dd, 2H, J 16.1 Hz, $\alpha \mathrm{H}), \delta 4.90$ (d, 2H, $J 32.2 \mathrm{~Hz}, \mathrm{H} 17), \delta 3.87\left(\mathrm{~s}, 3 \mathrm{H}, \mathrm{OCH}_{3}\right), \delta 2.13(\mathrm{~d}, 1 \mathrm{H}$, $J 10.6 \mathrm{~Hz}, \mathrm{H} 14), \delta 1.32$ (s, 3H, H18), $\delta 0.90$ (s, 3H, H20); ${ }^{13} \mathrm{C}$ NMR $\left(50 \mathrm{MHz}, \mathrm{CDCl}_{3}\right) \delta 191.0(\mathrm{C}=\mathrm{O}), \delta 176.95$ (C19), $\delta 163.92$ (C4), $\delta 156.19$ (C16), $\delta 130.07$ (C6), $\delta 130.07(\mathrm{C} 2), \delta 127.44(\mathrm{C} 1), \delta 113.99(\mathrm{C} 3), \delta 113.99$ (C5), $\delta 102.87$ (C17), $\delta 80.25$ (C13), $\delta 65.17(\alpha \mathrm{C}), \delta 56.98$ $(\mathrm{C} 5), \delta 55.51\left(\mathrm{OCH}_{3}\right), \delta 53.76(\mathrm{C} 9), \delta 47.40(\mathrm{C} 14), \delta 46.96$ (C15), $\delta 44.07$ (C4), $\delta 41.66$ (C8), $\delta 41.34$ (C7), $\delta 40.66$ (C1), $\delta 39.37$ (C10), $\delta 39.20$ (C12), $\delta 38.05$ (C3), $\delta 28.99$ (C18), $\delta 21.88$ (C6), $\delta 20.42$ (C11), $\delta 19.11$ (C2), $\delta 15.68$ (C20); ESI-MS $m / z$ calcd. for $\mathrm{C}_{29} \mathrm{H}_{38} \mathrm{O}_{5}$ : 489.27; found: $489.46[\mathrm{M}+\mathrm{Na}]^{+}$.

\section{General procedure for the preparation of hydrazones}

A solution of 4-nitrophenylhydrazine or 2,4-dinitrophenylhydrazine $(1.0 \mathrm{mmol})$ in concentrated $\mathrm{H}_{2} \mathrm{SO}_{4}(1 \mathrm{~mL})$, water $(1.5 \mathrm{~mL})$ ethanol $(1.5 \mathrm{~mL})$ was added to the solution $4 \mathbf{a}$ or $4 \mathbf{h}(0.5 \mathrm{mmol})$ in ethanol $(10 \mathrm{~mL})$ and the reaction mixture stirred for $12 \mathrm{~h}$ at $25{ }^{\circ} \mathrm{C}$. Water $(30 \mathrm{~mL})$ was then added and the product was recovered with EtOAc. The organic extract was dried over anhydrous sodium sulfate and the solvent was removed under reduced pressure. The residue was fractionated on silica rotors 
( $1 \mathrm{~mm}$, Chromatotron), and elution was made with suitable mixtures of acetone: $n$-hexane.

\section{Isosteviol isopropylhydrazone (4d)}

Isosteviol 16-hydrazone methyl ester (100 mg, $0.3 \mathrm{mmol}$ ) was refluxed with excess of acetone for $2 \mathrm{~h}$. A light yellow crystalline compound was isolated (83\%). IR (KBr) v / cm ${ }^{-1} 1721,1658,1452 ;{ }^{1} \mathrm{H}$ NMR (200 MHz, $\left.\mathrm{CDCl}_{3}\right) \delta 3.63\left(\mathrm{~s}, 3 \mathrm{H}, \mathrm{OCH}_{3}\right), \delta 2.68(\mathrm{dd}, 1 \mathrm{H}, J$ 18.4, $3.2 \mathrm{~Hz}, \mathrm{H} 15), \delta 2.01$ (s, 3H, H1'), $\delta 1.83$ (s, 3H, H3'), $\delta 1.17(\mathrm{~s}, 3 \mathrm{H}, \mathrm{H} 17), \delta 1.12(\mathrm{~s}, 3 \mathrm{H}, \mathrm{H} 18), \delta 0.69(\mathrm{~s}, 3 \mathrm{H}$, $\mathrm{H} 20) ;{ }^{13} \mathrm{C} \mathrm{NMR}\left(50 \mathrm{MHz}, \mathrm{CDCl}_{3}\right) \delta 177.9$ (C19), $\delta 174.3$ (C16), $\delta 159.2$ (C2'), $\delta 57.2$ (C5), $\delta 56.0$ (C14), $\delta 55.0$ (C9), $\delta 51.2\left(\mathrm{OCH}_{3}\right), \delta 44.2(\mathrm{C} 13), \delta 43.7(\mathrm{C} 4), \delta 41.1(\mathrm{C} 15)$, $\delta 40.6(\mathrm{C} 8), \delta 39.9(\mathrm{C} 1), \delta 39.4(\mathrm{C} 7), \delta 39.0(\mathrm{C} 12), \delta 38.0$ (C10), $\delta 37.9$ (C3), $\delta 28.8$ (C18), $\delta 24.9$ (C17), $\delta 22.2$ (C3'), $\delta 21.69$ (C6), $\delta 20.5$ (C11), $\delta 18.95$ (C2), $\delta 17.6$ $\left(\mathrm{C} 1^{\prime}\right.$ ), $\delta 13.25$ (C20); ESI-MS m/z calcd. for $\mathrm{C}_{24} \mathrm{H}_{39} \mathrm{~N}_{2} \mathrm{O}_{2}$ : 387.57; found: $387.36[\mathrm{M}+\mathrm{H}]^{+}$.

\section{Isosteviol 4-nitrophenylhydrazone (4e)}

Yellow crystalline solid; yield 56\%; IR $(\mathrm{KBr}) \mathrm{v} / \mathrm{cm}^{-1}$ 3319, 1693, 1595, 1322; ${ }^{1} \mathrm{H}$ NMR (200 MHz, $\mathrm{CDCl}_{3}$ ) $\delta 8.15$ (d, 2H, J 9.1 Hz, H3', H5'), $\delta 7.0$ (d, 2H, J $9.1 \mathrm{~Hz}$, H2', H6'), $\delta 2.96$ (d, 1H, J 17.0 Hz, H15), $\delta 1.3$ (s, 3H, $\mathrm{H} 17), \delta 1.19$ (s, 3H, H18), $\delta 0.89$ (s, 3H, H20); ${ }^{13} \mathrm{C}$ NMR $\left(50 \mathrm{MHz}, \mathrm{CDCl}_{3},\right) \delta 184.0(\mathrm{C} 19), \delta 163.5(\mathrm{C} 16), \delta 150.5$ (C4'), $\delta 139.6$ (C1'), $\delta 126.1$ (C3', C5'), $\delta 111.4$ (C2', C6'), $\delta 56.9$ (C5), $\delta 55.9$ (C14), $\delta 54.8$ (C9), $\delta 44.7$ (C13), $\delta 43.7$ (C4), $\delta 41.4(\mathrm{C} 8), \delta 41.1$ (C15), $\delta 39.7$ (C1), $\delta 39.4$ $(\mathrm{C} 7), \delta 38.3(\mathrm{C} 10), \delta 37.6(\mathrm{C} 3), \delta 36.6(\mathrm{C} 12), \delta 29.0(\mathrm{C} 18)$, $\delta 22.2(\mathrm{C} 17), \delta 21.6(\mathrm{C} 6), \delta 20.6(\mathrm{C} 11), \delta 18.8(\mathrm{C} 2), \delta 13.6$ (C20); ESI-MS $m / z$ calcd. for $\mathrm{C}_{26} \mathrm{H}_{34} \mathrm{~N}_{3} \mathrm{O}_{4}$ : 452.26; found: $452.33[\mathrm{M}-\mathrm{H}]^{-}$.

\section{7-Hydroxyisosteviol hydrazone (4k)}

Hydrazine hydrate $(2 \mathrm{~mL})$ was added into solution of 17-hydroxyisosteviol (100 mg, $0.3 \mathrm{mmol}$ ), in methanol $(10 \mathrm{~mL})$ and the mixture was refluxed for 8 hours. The solvent and excess of hydrazine were removed under reduced pressure and the product was recrystallized from methanol (90 mg, 86\%); IR (KBr) v / cm-1 3412, 3221, 1698, 1175; ${ }^{1} \mathrm{H}$ NMR (200 MHz, CD 3 OD) $\delta 3.57$ (s, 2H, H17), $\delta 2.86$ (d, 1H, J 17.5 Hz, H12), $\delta 1.18$ (s, 3H, H18), $\delta 0.96$ (s, 3H, H20); $\left.{ }^{13} \mathrm{C} \mathrm{NMR} \mathrm{(50} \mathrm{MHz,} \mathrm{CD} \mathrm{OD}\right), \delta 182.9$ (C19), $\delta 165.3$ (C16), $\delta 66.1$ (C17), $\delta 57.4$ (C5), $\delta 55.6$ (C9), $\delta 51.0$ (C14), $\delta 49.1$ (C13), $\delta 44.1$ (C4), $\delta 41.2$ (C15), $\delta 40.8$ (C8), $\delta 40.2(\mathrm{C} 1), \delta 38.6$ (C3), $\delta 38.0$ (C10), $\delta 36.8(\mathrm{C} 7), \delta 34.2(\mathrm{C} 12), \delta 28.8(\mathrm{C} 18), \delta 21.9(\mathrm{C} 6), \delta 19.6$ (C11), $\delta 19.2$ (C2), $\delta 12.9$ (C20); ESI-MS m/z calcd. for $\mathrm{C}_{20} \mathrm{H}_{33} \mathrm{~N}_{2} \mathrm{O}_{3}$ : 349.24; found: $349.30[\mathrm{M}+\mathrm{H}]^{+}$.
17-Hydroxyisosteviol, 4-nitrophenylhydrazone (4I)

Yellow solid; yield 56\%; IR (KBr) v / $\mathrm{cm}^{-1} 3315,1692$, 1618, 1337; ${ }^{1} \mathrm{H}$ NMR $\left(200 \mathrm{MHz}\right.$, acetone- $\left.d_{6}\right) 9.1(\mathrm{~s}, 1 \mathrm{H}$, NH), 8.09 (d, 2H, J 9.36 Hz, H3', H5'), $\delta 7.15$ (d, 2H, $J 9.33$ Hz, H2', H6'), $\delta 3.6$ (d, 1H, J 7.4 Hz, H17), 3.7 (d, $1 \mathrm{H}, J 7.4 \mathrm{~Hz}, \mathrm{H} 17) \delta 2.97$ (dd, 1H, J 18.23, $2.86 \mathrm{~Hz}, \mathrm{H} 15)$, $\delta 1.21$ (s, 3H, H18), 0.87 (s, 3H, H20); ${ }^{13} \mathrm{CNMR}(50 \mathrm{MHz}$, acetone- $\left.d_{6}\right) \delta 178.1$ (C19), $\delta 162.7$ (C16), $\delta 151.5$ (C4'), $\delta 138.8\left(\mathrm{C}^{\prime}\right.$ '),$\delta 125.6\left(\mathrm{C}^{\prime}, \mathrm{C} 5\right.$ '), $\delta 111.1$ (C2', C6'), $\delta 65.9$ (C17), $\delta 56.7$ (C5), $\delta 55.3$ (C9), $\delta 50.7$ (C14), $\delta 50.0(\mathrm{C} 13), \delta 43.2(\mathrm{C} 4), \delta 41.1(\mathrm{C} 8), \delta 40.9(\mathrm{C} 15), \delta 39.7$ (C1), $\delta 38.0$ (C10), $\delta 37.9$ (C3), $\delta 37.8$ (C7), $\delta 34.5$ (C12), $\delta 28.4$ (C18), $\delta 21.7$ (C6), $\delta 19.8$ (C11), $\delta 18.8(\mathrm{C} 2), \delta 13.1$ (C20); ESI-MS $m / z$ calcd. for $\mathrm{C}_{26} \mathrm{H}_{33} \mathrm{~N}_{3} \mathrm{O}_{5}$ : 468.26; found: $468.37[\mathrm{M}-\mathrm{H}]^{-}$.

\section{7-Hydroxyisosteviol-2,4-dinitrophenylhydrazone (4m)}

Yellow solid; yield 55\%; IR (KBr) v / $\mathrm{cm}^{-1} 3446,3315$, 1692, 1618, 1337; ${ }^{1} \mathrm{H}$ NMR (200 MHz, acetone- $\left.d_{6}\right) \delta 10.7$ (s, 1H, NH), $\delta 8.99$ (d, 1H, J 2.5 Hz, H3'), $\delta 8.19$ (dd, 1H, $J$ 9.6, $2.51 \mathrm{~Hz}, \mathrm{H} 5$ '), $\delta 7.7$ (d, 1H, J 9.6 Hz, H6'), $\delta 3.78$ (dd, 1H, J 19.2, $11.4 \mathrm{~Hz}, \mathrm{H} 17), \delta 2.96$ (dd, 1H, J 18.2, $2.86 \mathrm{~Hz}, \mathrm{H} 15), \delta 1.3$ (s, 3H, H18), $\delta 0.94$ (s, 3H, H20); ${ }^{13} \mathrm{C}$ NMR (50 MHz, acetone- $\left.d_{6}\right) \delta 183.7$ (C19), $\delta 170.90$ (C16), $\delta 144.5$ (C4'), $\delta 137.7$ (C2'), $\delta 129.8$ (C3'), $\delta 128.8$ (C1'), $\delta 123.3$ (C5'), $\delta 115.8$ (C6'), $\delta 66.3$ (C17), $\delta 56.8$ (C5), $\delta 55.4$ (C9), $\delta 50.6$ (C13), $\delta 50.5$ (C14), $\delta 43.6(\mathrm{C} 4)$, $\delta 41.5(\mathrm{C} 8), \delta 40.6(\mathrm{C} 15), \delta 39.6(\mathrm{C} 1), \delta 38.3$ (C10), $\delta 37.9$ (C3), $\delta 37.6(\mathrm{C} 7), \delta 34.3$ (C12), $\delta 28.9$ (C18), $\delta 21.53$ (C6), $\delta 19.7$ (C11), $\delta 18.7$ (C2), $\delta 12.9$ (C20); ESI-MS m/z calcd. for $\mathrm{C}_{26} \mathrm{H}_{33} \mathrm{~N}_{4} \mathrm{O}_{7}:$ 513.24; found: $513.24[\mathrm{M}-\mathrm{H}]^{-}$.

\section{Isosteviol p-methoxyphenacyl ester (4n)}

White solid; yield 81\%; IR (KBr) v / $\mathrm{cm}^{-1} 2953,1733$, 1694, 1598, 1144; ${ }^{1} \mathrm{H}$ NMR $\left(200 \mathrm{MHz}, \mathrm{CDCl}_{3}\right) \delta 7.89$ (d, 2H, J 9.0 Hz, H2', H6'), $\delta 6.94$ (d, 2H, J 9.0 Hz, H3', $\mathrm{H}^{\prime}$ '), $\delta 5.2(\mathrm{~d}, 1 \mathrm{H}, J 16.0 \mathrm{~Hz}, \alpha \mathrm{H}), 5.3(\mathrm{~d}, 1 \mathrm{H}, J 16.0 \mathrm{~Hz}$, $\alpha \mathrm{H}), \delta 3.87\left(\mathrm{~s}, 3 \mathrm{H}, \mathrm{OCH}_{3}\right), \delta 2.65(\mathrm{dd}, 1 \mathrm{H}, J 18.7,3.65 \mathrm{~Hz}$, H15), $\delta 1.33$ (s, 3H, H17), $\delta 0.97$ (s, 3H, H18), $\delta 0.77$ (s, $3 \mathrm{H}, \mathrm{H} 20) ;{ }^{13} \mathrm{C} \mathrm{NMR}\left(50 \mathrm{MHz}, \mathrm{CDCl}_{3}\right) \delta 190.93$ (C=O), $\delta 176.80$ (C19), $\delta 163.95$ (C4'), $\delta 130.05$ (C2', C6'), $\delta 127.44\left(\mathrm{C}^{\prime}\right.$ '),$\delta 114.00(\mathrm{C} 3$ ', C5'), $\delta 65.17(\alpha \mathrm{C}), \delta 57.14$ $(\mathrm{C} 5), \delta 55.50\left(\mathrm{OCH}_{3}\right), \delta 54.75(\mathrm{C} 9), \delta 54.30(\mathrm{C} 14), \delta 48.69$ (C13), $\delta 48.48$ (C15), $\delta 44.07$ (C4), $\delta 41.53$ (C7), $\delta 39.82$ (C1), $\delta 39.46(\mathrm{C} 8), \delta 38.09$ (C10), $\delta 38.01$ (C12), $\delta 37.30$ (C3), $\delta 29.10$ (C18), $\delta 21.63$ (C6), $\delta 20.32$ (C11), $\delta 19.84$ (C17), $\delta 18.93$ (C2), $\delta 13.55$ (C20); ESI-MS m/z calcd. for $\mathrm{C}_{29} \mathrm{H}_{38} \mathrm{O}_{5} \mathrm{Na}$ : 466.27; found: $466.59[\mathrm{M}+\mathrm{Na}]^{+}$.

Isosteviol oxime p-methoxyphenacyl ester (40) Amorphous white solid; yield 78\%; IR (KBr) $v / \mathrm{cm}^{-1}$ 
3446, 2949, 1723, 1690, 1599, 1159; ${ }^{1} \mathrm{H}$ NMR $(200 \mathrm{MHz}$, $\left.\mathrm{CDCl}_{3}\right) \delta 7.89\left(\mathrm{~d}, 2 \mathrm{H}, J 8.9 \mathrm{~Hz}, \mathrm{H} 2^{\prime}, \mathrm{H} 6\right.$ '),$\delta 6.95(\mathrm{~d}, 1 \mathrm{H}$, $J 8.91 \mathrm{~Hz}, \mathrm{H} 3$ ', H5'), $\delta 5.23(\mathrm{~d}, 1 \mathrm{H}, J 16.0 \mathrm{~Hz}, \alpha \mathrm{H}), \delta 3.87$ $\left(\mathrm{s}, 3 \mathrm{H}, \mathrm{OCH}_{3}\right), \delta 2.98(\mathrm{dd}, 1 \mathrm{H}, J 18.8,3.00 \mathrm{~Hz}, \mathrm{H} 15), \delta 1.33$ (s, 3H, H17), $\delta 1.09$ (s, 3H, H18), $\delta 0.82$ (s, 3H, H20); ${ }^{13} \mathrm{C}$ NMR $\left(50 \mathrm{MHz}, \mathrm{CDCl}_{3}\right) \delta 190.93(\mathrm{C}=\mathrm{O}), \delta 177.00$ (C19), $\delta 170.45$ (C16), $\delta 163.92$ (C4'), $\delta 130.06$ (C2', C6'), $\delta 127.44\left(\mathrm{C} 1^{\prime}\right), \delta 113.99\left(\mathrm{C}^{\prime}, \mathrm{C}^{\prime}{ }^{\prime}\right), \delta 65.16(\alpha \mathrm{C}), \delta 57.14$ (C5), $\delta 56.28(\mathrm{C} 14), \delta 55.49\left(\mathrm{OCH}_{3}\right), \delta 54.90(\mathrm{C} 9), \delta 44.06$ (C13), $\delta 43.72$ (C4), $\delta 40.90$ (C15), $\delta 40.63(\mathrm{C} 8), \delta 39.92$ (C1), $\delta 39.45$ (C7), $\delta 38.13$ (C10), $\delta 38.06$ (C3), $\delta 36.78$ (C12), $\delta 29.04$ (C18), $\delta 22.12$ (C17), $\delta 21.67$ (C6), $\delta 20.42$ (C11), $\delta 18.95$ (C2), $\delta 13.55$ (C20); ESI-MS $m / z$ calcd. for $\mathrm{C}_{29} \mathrm{H}_{39} \mathrm{NO}_{5}$ : 481.28 ; found: $481.45[\mathrm{M}]^{+}$.

\section{6-Hydroxyisosteviol p-methoxyphenacyl ester (4p)}

Amorphous white solid; yield 75\%; IR (KBr) v / $\mathrm{cm}^{-1}$ 3556, 2958, 1723, 1690, 1603, 1159; ${ }^{1} \mathrm{H}$ NMR $(200 \mathrm{MHz}$, $\left.\mathrm{CDCl}_{3}\right) \delta 7.90\left(\mathrm{~d}, 2 \mathrm{H}, J 8.9 \mathrm{~Hz}, \mathrm{H} 2^{\prime}, \mathrm{H}^{\prime}\right), \delta 6.95(\mathrm{~d}, 1 \mathrm{H}$, $J 8.9 \mathrm{~Hz}, \mathrm{H} 3$ ', H5'), $\delta 5.2(\mathrm{~d}, 1 \mathrm{H}, J 16.1 \mathrm{~Hz}, \alpha \mathrm{H}), \delta 5.3$ $(\mathrm{d}, 1 \mathrm{H}, J 16.1 \mathrm{~Hz}, \alpha \mathrm{H}), \delta 3.87\left(\mathrm{~s}, 3 \mathrm{H}, \mathrm{OCH}_{3}\right), \delta 1.31$ (s, 3H, H17), $\delta 0.91$ (s, 3H, H18), $\delta 0.80$ (s, 3H, H20); ${ }^{13} \mathrm{C}$ NMR $\left(50 \mathrm{MHz}, \mathrm{CDCl}_{3}\right) \delta 191.08(\mathrm{C}=\mathrm{O}), \delta 177.03$ (C19), $\delta 163.88$ (C4'), $\delta 130.04$ (C2', C6'), $\delta 127.50$ (C1'), $\delta 113.95\left(\mathrm{C}^{\prime}, \mathrm{C} 5{ }^{\prime}\right), \delta 80.52(\mathrm{C} 16), \delta 65.17(\alpha \mathrm{C}), \delta 57.22$ (C5), $\delta 55.85$ (C9), $\delta 55.47\left(\mathrm{OCH}_{3}\right), \delta 55.30(\mathrm{C} 14), \delta 44.04$ (C4), $\delta 42.84$ (C15), $\delta 42.07$ (C13), $\delta 41.99$ (C8), $\delta 41.75$ (C7), $\delta 39.91$ (C1), $\delta 38.11$ (C3), $\delta 38.09$ (C10), $\delta 33.71$ (C12), $\delta 29.12$ (C18), $\delta 24.88$ (C17), $\delta 21.69$ (C6), $\delta 20.45$ (C11), $\delta 18.95$ (C2), $\delta 13.47$ (C20); ESI-MS $m / z$ calcd. for $\mathrm{C}_{29} \mathrm{H}_{40} \mathrm{O}_{5} \mathrm{Na}$ : 491.29; found: $491.35[\mathrm{M}+\mathrm{Na}]^{+}$.

\section{Isosteviol benzyl ester (4q)}

To the solution of isosteviol $(318 \mathrm{mg}, 1 \mathrm{mmol})$, in acetone $(40 \mathrm{~mL})$, potassium carbonate $(200 \mathrm{mg}$, $1.45 \mathrm{mmol})$, and benzyl chloride ( $2 \mathrm{~mL})$, were added and the mixture was refluxed for two hours. After filtration the solvent and excess benzyl chloride were removed under reduced pressure. A white crystalline solid was recovered (340 mg, 83\%); IR (KBr) v/ $\mathrm{cm}^{-1} 1738,1720,1454,1147$, 694; ' $\mathrm{H}$ NMR (200 MHz, $\left.\mathrm{CDCl}_{3}\right) \delta 7.34$ (s, 5H, H'-H6'), 5.08 (dd, $1 \mathrm{H}, J$ 18.1, $12.4 \mathrm{~Hz}, \mathrm{H \alpha}$ ), $\delta 2.55$ (dd, $1 \mathrm{H}, J$ 18.1, $12.4 \mathrm{~Hz}, \mathrm{H} 15), \delta 1.21$ (s, 3H, H17), 0.96 (s, 3H, H18), $\delta 0.6$ (s, 3H, H20); $\mathrm{C}^{13} \mathrm{NMR}\left(50 \mathrm{MHz}, \mathrm{CDCl}_{3}\right) \delta 176.9$ (C19), $\delta 135.9$ (C1'), $\delta 128.4$ (C3', C5'), $\delta 128.35$ (C2', C6'), $\delta 128.1$ (C4'), $\delta 66.1(\alpha \mathrm{C}), \delta 57.1$ (C5), $\delta 54.6$ (C9), $\delta 54.2$ (C14), $\delta 48.6$ (C13), $\delta 48.3$ (C15), $\delta 43.8$ (C4), $\delta 41.4$ (C7), $\delta 39.7$ (C1), $\delta 39.4$ (C8), $\delta 37.9$ (C10, C12), $\delta 37.3(\mathrm{C} 3), \delta 28.9(\mathrm{C} 18), \delta 21.7(\mathrm{C} 6), \delta 20.3(\mathrm{C} 11), \delta 19.8$ (C17), $\delta 18.89(\mathrm{C} 2), \delta 13.3$ (C20); ESI-MS $m / z$ calcd. for $\mathrm{C}_{27} \mathrm{H}_{37} \mathrm{O}_{3}: 409.27$; found: $409.30[\mathrm{M}+\mathrm{H}]^{+}$.

\section{Antitrypanosomal bioassay}

Vero cells (ATCC CCL-81) were maintained at $37{ }^{\circ} \mathrm{C}$ in a humidified 5\% $\mathrm{CO}_{2}$ atmosphere. The cells were grown in $75 \mathrm{~cm}^{2}$ culture flasks with RPMI-1640 medium supplemented with $10 \%$ fetal calf serum (FCS), $1 \% \mathrm{~L}$-glutamine, $1 \%$ penicillin and $10 \mathrm{mg} \mathrm{mL}^{-1}$ streptomycin. For the cytotoxicity bioassays, 4-day-old confluent Vero cells monolayers were washed with phosphate buffered saline (PBS, pH 7.2) and detached from the substrate by treatment with $0.25 \%$ trypsin $+0.1 \%$ EDTA for 5 minutes at $37^{\circ} \mathrm{C}$. The cells were then resuspended in the same medium, centrifuged for 2 minutes at $100 \mathrm{~g}$ and the cell pellet was collected.

Culture epimastigote forms of Trypanosoma cruzi clone Dm28c were maintained in LIT (liver infusion-tryptose) medium at $28{ }^{\circ} \mathrm{C}$ with serial passagesat every three days (mid-log phase of growth). ${ }^{42}$ For the experiments, parasites obtained from 72-hour cultures were inoculated into fresh LIT medium and then added to 96-well plates at a concentration of $5 \times 10^{7}$ cells per well.

To obtain cell-derived trypomastigote forms, Vero cell cultures were infected with trypomastigote forms at a 10:1 parasite:host cell ratio. After four hours of interaction, the host cell monolayers were washed with PBS to remove noninternalized parasites. The cultures were kept for 96 hours at $37{ }^{\circ} \mathrm{C}$ in $\mathrm{RPMI} / 2.5 \% \mathrm{FCS}$ in a $5 \% \mathrm{CO}_{2}$ humidified atmosphere. After that period, trypomastigotes released to the supernatant were collected and washed with PBS by centrifugation at $3000 \mathrm{~g}$. The purified trypomastigotes were transferred to RPMI-1640 medium at a concentration of $5 \times 10^{7}$ cells $\mathrm{mL}^{-1}$ and then added to 96 -well plates at $100 \mu \mathrm{L}$ per well.

For the assays steviol and isosteviol derivatives were diluted in dimethylsulfoxide (DMSO) and added to 96 well plates containing parasites (epimastigotes or trypomastigotes), at final concentrations ranging from 10 to $600 \mu \mathrm{M}$. The $50 \%$ inhibitory concentration after 24 hours of incubation $\left(\mathrm{IC}_{50}\right.$ per $24 \mathrm{~h}$ ) was determined with the cell viability marker MTT [3-(4,5-dimethylthiazol2-yl)-2,5-diphenyltetrazolium bromide], according to standard protocols. ${ }^{43}$ The assays were quantified at $550 \mathrm{~nm}$ using the ELISA reader BIOTEXEL-800 (Biotek, Winooski, VT, USA). All experiments were performed in biological and technical triplicate. The CompuSyn software was used to calculate the $\mathrm{IC}_{50}$ per $24 \mathrm{~h}$. Controls were grown in medium containing $0.5 \% \mathrm{DMSO}$, without addition of derivates.

For the cytotoxicity assays Vero cells were seeded at a concentration of $2 \times 10^{4}$ cells per well in 96-well plates, containing RPMI-1640 medium supplemented with $10 \%$ FCS, and maintained at $37{ }^{\circ} \mathrm{C}$ and $5 \% \mathrm{CO}_{2}$ atmosphere. 
After 24 hours of cultivation, oxime of isosteviol (10 to $75 \mu \mathrm{M}$ ) or benznidazole (3.8 to $4000 \mu \mathrm{M}$ ) were added. The cytotoxic concentration $\left(\mathrm{CC}_{50}\right.$ per $\left.24 \mathrm{~h}\right)$ was determined after $24 \mathrm{~h}$ of incubation, using the MTT enzymatic assay as described above. The selectivity index (SI) was determined based on the ratio of the $\mathrm{CC}_{50}$ value in the host cell divided by the $\mathrm{IC}_{50}$ value of the parasite.

\section{Antimalarial bioassay}

The antimalarial activity was evaluated by $\left[{ }^{3} \mathrm{H}\right]$-hypoxanthine incorporation ${ }^{44}$ and lactate dehydrogenase ${ }^{45}$ assays. The compounds were diluted in DMSO (50 $\left.\mathrm{mg} \mathrm{mL}^{-1}\right)$ and serial dilutions (1.56-50 $\mathrm{g} \mathrm{mL}^{-1}$ ) were prepared in RPMI-1640 medium. The assay was carried out in 96-well plates using suspension of erythrocytes infected with $P$. falciparum $\mathrm{W} 2$, chloroquineresistant (ring-stage parasites synchronized in sorbitol, $2 \%$ hematocrit, $1 \%$ parasitemia). Controls with infected or noninfected erythrocytes were used. The results were analyzed as sigmoidal dose-response curves and the $\mathrm{IC}_{50}$ values (concentration in that the growth of the parasites is 50\% inhibited) of three independent experiments were obtained. The results were classified in accordance to a standard criteria: very active $\left(\mathrm{IC}_{50}<1 \mu \mathrm{g} \mathrm{mL} \mathrm{m}^{-1}\right)$, active $\left(\mathrm{IC}_{50} 1-15 \mu \mathrm{g} \mathrm{mL} \mathrm{m}^{-1}\right.$ ), moderately active $\left(\mathrm{IC}_{50} 15.1-25 \mu \mathrm{g} \mathrm{mL}^{-1}\right)$, slightly active $\left(\mathrm{IC}_{50} 25.1-50 \mu \mathrm{g} \mathrm{mL}^{-1}\right)$ and inactive $\left(\mathrm{IC}_{50}>50 \mu \mathrm{g} \mathrm{mL}^{-1}\right)$.

The cytotoxicity of the most active compounds, $2 \mathbf{c}$ and $\mathbf{2} \mathbf{j}$, was evaluated against human hepathoblastoma HepG2 A16 cell line ${ }^{46}$ using MTT assay. ${ }^{47}$ The results were expressed as $\mathrm{CC}_{50}$ values (cytotoxic concentration that inhibit 50\% the cell growth) of three independent experiments. Selectivity index (SI) was calculated by the $\mathrm{CC}_{50}$ value for HepG2 A16 cells divided by $\mathrm{IC}_{50}$ value for P. falciparum.

\section{Supplementary Information}

Spectroscopic spectra of compounds and further bioassays data are available free of charge at http://jbcs.sbq.org.br as PDF file.

\section{Acknowledgments}

The authors are grateful to Coordenação de Aperfeiçoamento de Pessoal de Nível Superior (CAPES) and Conselho Nacional de Desenvolvimento Científico e Tecnológico/Third World Academy of Sciences (CNPq/ TWAS) for the financial support. We are also grateful to PRONEX, Rede Malária, CNPq FAPEMIG (Brazil) and its coordinator, Prof Alaide B. de Oliveira, for the bioassays.

\section{References}

1. World Health Organization (WHO); World Malaria Report: 2013; WHO Press: Geneva, 2013.

2. Coordenação Geral do Programa Nacional de Controle da Malária (CGPNCM); Brasília, 2013. Available at http:// portalsaude.saude.gov.br/images/pdf/2014/maio/30/boletimepidemiol--gico-1-de-2013-malaria.pdf, accessed in January 2016.

3. Chugh, M.; Scheurer, C.; Sax, S.; Bilsland, E.; van Schalkwyk, D. A.; Wicht, K. J.; Hofmann, N.; Sharma, A.; Bashyam, S.; Singh, S.; Oliver, S. G.; Egan, T. J.; Malhotra, P.; Sutherland, C. J.; Beck, H.-P.; Wittlin, S.; Spangenberg, T.; Ding, X. C.; Antimicrob. Agents Chemother. 2015, 59, 1110.

4. Batista, R.; Silva Ade Jr., J.; de Oliveira, A. B.; Molecules 2009 , 14, 3037.

5. Nogueira, C. R.; Lopes, L. M. X.; Molecules 2011, 16, 2146.

6. Bringmann, G.; Saeb, W.; Assi, L. A.; Francois, G.; Sankara Narayanan, A. S.; Peters, K.; Peters, E. M.; Planta Med. 1997, 63, 255.

7. Attioua, B.; Weniger, B.; Chabert, P.; Pharm. Biol. 2007, 45, 263.

8. Domínguez-Carmona, D. B.; Escalante-Erosa, F.; García-Sosa, K.; Ruiz-Pinell, G.; Gutierrez-Yapu, D.; Chan-Bacab, M. J.; Moo-Puc, R. E.; Veitch, N. C.; Giménez-Turba, A.; PeñaRodríguez, L. M.; J. Braz. Chem. Soc. 2011, 22, 1279.

9. Duker-Eshun, G.; Jaroszewski, J. W.; Asomaning, W. A.; Oppong-Boachie, F.; Brogger Christensen, S.; Phytother. Res. 2004, 18, 128.

10. Lenta, B. N.; Devkota, K. P.; Ngouela, S.; Fekam-Boyom, F.; Naz, Q.; Choudhary, M. I.; Tsamo, E.; Rosenthal, P. J.; Sewald, N.; Chem. Pharm. Bull. 2008, 56, 222.

11. Lenta, B. N.; Ngouela, S.; Fekam-Boyom, F.; Tantangmo, F.; Feuya-Tchouya, G. R.; Tsamo, E.; Gut, J.; Rosenthal, P. J.; Donald-Connolly, J.; Chem. Pharm. Bull. 2007, 55, 464.

12. Zofou, D.; Kowa, T. K.; Wabo, H. K.; Ngemenya, M. N.; Tane, P.; Titanji, V. P.; Malar. J. 2011, 10, 167.

13. da Silva, G. N.; Maria, N. R.; Schuck, D. C.; Cruz, L. N.; de Moraes, M. S.; Nakabashi, M.; Graebin, C.; Gosmann, G.; Garcia, C. R.; Gnoatto, S. C.; Malar. J. 2013, 12, 89.

14. de Sa, M. S.; Costa, J. F.; Krettli, A. U.; Zalis, M. G.; Maia, G. L.; Sette, I. M.; Camara, C. A.; Filho, J. M.; Giulietti-Harley, A. M.; Ribeiro dos Santos, R.; Soares, M. B.; Parasitol. Res. 2009, 105, 275.

15. Domínguez-Carmona, D. B.; Escalante-Erosa, F.; García-Sosa, K.; Ruiz-Pinell, G.; Gutierrez-Yapu, D.; Chan-Bacab, M. J.; Giménez-Turba, A.; Peña-Rodríguez, L. M.; Phytomedicine 2010, 17, 379.

16. Graziose, R.; Rojas-Silva, P.; Rathinasabapathy, T.; Dekock, C.; Grace, M. H.; Poulev, A.; Ann Lila, M.; Smith, P.; Raskin, I.; J. Ethnopharmacol. 2012, 142, 456. 
17. Innocente, A. M.; Silva, G. N.; Cruz, L. N.; Moraes, M. S.; Nakabashi, M.; Sonnet, P.; Gosmann, G.; Garcia, C. R.; Gnoatto, S. C.; Molecules 2012, 17, 12003.

18. Ma, C. Y.; Musoke, S. F.; Tan, G. T.; Sydara, K.; Bouamanivong, S.; Southavong, B.; Soejarto, D. D.; Fong, H. H.; Zhang, H. J.; Chem. Biodivers. 2008, 5, 2442.

19. Suksamrarn, A.; Tanachatchairatana, T.; Kanokmedhakul, S.; J. Ethnopharmacol. 2003, 88, 275.

20. Suksamrarn, S.; Panseeta, P.; Kunchanawatta, S.; Distaporn, T.; Ruktasing, S.; Suksamrarn, A.; Chem. Pharm. Bull. 2006, 54, 535 .

21. van Wyk, A. W. W.; Lobb, K. A.; Caira, M. R.; Hoppe, H. C.; Davies-Coleman, M. T.; J. Nat. Prod. 2007, 70, 1253.

22. Mthembu, X. S.; van Heerden, F. R.; Fouche, G.; S. Afr. J. Bot. 2010, 76, 82.

23. Uys, A. C. U.; Malan, S. F.; van Dyk, S.; van Zyl, R. L.; Bioorg. Med. Chem. Lett. 2002, 12, 2167.

24. World Health Organization (WHO); Chagas Disease, Fact Sheet 340; WHO Press: Geneva, 2015. Available at http://www.who. int/mediacentre/factsheets/fs340/en/, accessed in January 2016.

25. Coura, J. R.; Mem. Inst. Oswaldo Cruz 2009, 104, 549.

26. Campos, M. C. O.; Leon, L. L.; Taylor, M. C.; Kelly, J. M.; Mol. Biochem. Parasitol. 2014, 193, 17.

27. Takahashi, J. A.; Vieira, H. S.; Silva, E. A.; Boaventura, M. A. D.; Oliveira, A. B.; Chiari, E.; Braz. J. Pharmacog. 2002, 12,118 .

28. Vieira, H. S.; Takahashi, J. A.; de Oliveira, A. B.; Chiari, E.; Boaventura, M. A. D.; J. Braz. Chem. Soc. 2002, 13, 151.

29. do Nascimento, A. M.; Chaves, J. S.; Albuquerque, S.; de Oliveira, D. C. R.; Fitoterapia 2004, 75, 381.

30. Haraguchi, S. K.; Silva, A. A.; Vidotti, G. J.; dos Santos, P. V.; Garcia, F. P.; Pedroso, R. B.; Nakamura, C. V.; de Oliveira, C. M. A.; da Silva, C. C.; Molecules 2011, 16, 1166.

31. Rojas Ruiz, F. A.; Garcia-Sanchez, R. N.; Estupinan, S. V.; Gomez-Barrio, A.; Torres Amado, D. F.; Perez-Solorzano, B. M.; Nogal-Ruiz, J. J.; Martinez-Fernandez, A. R.; Kouznetsov, V. V.; Bioorg. Med. Chem. 2011, 19, 4562.
32. Boakye Yiadom, K.; Fiagbe, N. I.; Ayim, J. S.; Lloydia 1977, 40, 543.

33. Baratto, L. C.; Porsani, M. V.; Pimentel, I. C.; Pereira-Netto, A. B.; Paschke, R.; Oliveira, B. H.; Eur. J. Med. Chem. 2013, $68,121$.

34. Oliveira, B. H.; Sant'Ana, A. E. G.; Bastos, D. Z. L.; Phytochem. Anal. 2002, 13, 368.

35. Takahashi, J. A.; Boaventura, M. A. D.; Bayma, J. D.; Oliveira, A. B.; Phytochemistry 1995, 40, 607.

36. Ogawa, T.; Nozaki, M.; Matsui, M.; Tetrahedron 1980, 36, 2641.

37. Avent, A. G.; Hanson, J. R.; de Oliveira, B. H.; Phytochemistry 1990, 29, 2712.

38. Ali, M. S.; Hanson, J. R.; de Oliveira, B. H.; Phytochemistry 1992, 31, 507.

39. Al'fonsov, V. A.; Bakaleinik, G. A.; Gubaidullin, A. T.; Kataev, V. E.; Kovylyaeva, G. I.; Konovalov, A. I.; Litvinov, I. A.; Strobykina, I. Y.; Strobykin, S. I.; Zh. Obshch. Khim. 1998, 68, 1813.

40. Al'fonsov, V. A.; Andreeva, O. V.; Bakaleinik, G. A.; Beskrovnyi, D. V.; Gubaidullin, A. T.; Kataev, V. E.; Kovylyaeva, G. I.; Konovalov, A. I.; Korochkina, M. G.; Litvinov, I. A.; Militsina, O. I.; Strobykina, I. Y.; Russ. J. Gen. Chem. 2003, 73, 1255.

41. Silva, F. O.; Ferraz, V.; Talanta 2006, 68, 643.

42. Camargo, O. P.; Rev. Inst. Med. Trop. Sao Paulo 1961, 6, 93.

43. Francis, D.; Rita, L.; J. Immunol. Meth. 1986, 89, 271.

44. Desjardins, R. E.; Canfield, C. J.; Haynes, J. D.; Chulay, J. D.; Antimicrob. Agents Chemother. 1979, 16, 710.

45. Makler, M. T.; Hinrichs, D. J.; Am. J. Trop. Med. Hyg. 1993, 48, 205.

46. Varotti, F. P.; Botelho, A. C. C.; Andrade, A. A.; de Paula, R. C.; Fagundes, E. M. S.; Valverde, A.; Mayer, L. M. U.; Mendonça, J. S.; de Souza, M. V. N.; Boechat, N.; Krettli, A. U.; Antimicrob. Agents Chemother. 2008, 52, 3868.

47. Denizot, F.; Lang, R.; J. Immunol. Methods 1986, 89, 271.

Submitted: September 5, 2015 Published online: January 27, 2016 